La presenza nell' acqua di tracce d'alcune sostanze (le coloranti, ad es.) influisce moltissimo sull' intensitá e talvolta sul segno della elettrizzazione.

Nello sviluppo di elettricità per contatto di aria ed acqua, occorre o la lacerazione di una bolla d'aria o lo spruzzamento di una goccia d'acqua. Holmgren ${ }^{1}$ ) osservò che il semplice soffio dell' aria sulla superficie dell' acqua non produce elettricità, mentre questa si sviluppa con un'agitazione meccanica violenta della massa liquida.

Per dimostrare in altro modo questo medesimo principio soffiai l' aria attraverso sostanze porose (carta da filtro, tela, cotone, lana) imbevute d'acqua senza mai osservare il minimo sviluppo di elettricità ${ }^{2}$ ).

Roma, 15 Maggio 1902.

\title{
SULL' USO DEL RETICOLO DI DIFFRAZIONE NELLO STUDIO DELLO SPETTRO ULTRAVIOLETTO
}

del Dott. R. MAGINI ${ }^{3}$ ).

(Sunto della Not. inserita nei Rend. della R. Acc. dei Lincei, Vol, 11, 2. sem., 1902).

1. In un lungo lavoro che ho ancora in corso sul campo spettrale ultravioletto, ho avuto occasione di portare delle modificazioni all' ordinario metodo di uso del reticolo di Rowland per lo studio dei raggi più refrangibili.

Credo utile pertanto darne una breve relazione, in attesa della pubblicazione dell' intero lavoro.

Io avevo bisogno di uno spettro molto intenso $e$ molto ricco di righe e di bande.

La prima di queste necessitã si presenta nella maggiop parte dei lavori con lo spettro ultravioletto, perchè soltanto

1) Sur le développement de l'électr. au contact de l'air et de l'eau. Soe. physiog. de Jund 1894.

2) Qnesto lavoro fu eseguito nel R. Istituto Fisico di Roma con l'aiuto e la direzione dei Proff. Blaserna e Sella ai quali l' autore porge i più sentiti ringrazianmenti.

3) Laroto eseguito nell' Istituto di Fisica della R. Università di Pisa, diretto dal Prof. A. Battelli. 
allora riesce facile e rapido l' esame dei raggi d'onda piủ corta, quasi sempre deboli e facilnente assorbibili dai mezzi interposti.

La seconda condizione, cui non è possibile soddisfare se non osservando sovrapposti gli spettri emessi contemporaneamente da vari corpi, conduce ad una grande complessità di righe e di bande e richiede quindi una grande dispersione affinchè riesca poi possibile distinguere e misurare gli elementi di quei complessi che differiscono per frazioni piccolissime di lunghezza d'onda. Per questa ragione non è allora possibile adoperare lo spettro ultravioletto del primo ordine che, pur essendo sufficientemente luminoso, possiede una dispersione troppo debole.

Lo spettro del secondo ordine, cui bisognerebbe allora ricorrere, presenta altri difetti : è poco luminoso e gli si sovrappongono spettri di altri ordiní cioè da una parte lo spettro luminoso del primo ordine a cominciare dal violetto e dall'altra l' estremo ultravioletto del terzo.

La presenza di questi spettri rende penosa l'interpreta. zione delle osservazioni e ne maschera i risultati ; bisognerebbe allora procedere alla separazione mediante prismi di quarzo. In tal modo però può venire a mancare la ragione che indusse ad adoperare il reticolo di diffrazione, cioè quella di eliminare ogni mezzo assorbente.

Tali essendo i termini del problema, espongo subito il modo come l' ho risoluto.

2. Il reticolo da me adoperato ha un raggio di curvatura di 3 metri e 14438 linee per pollice.

La sua installazione è semplice e non differisce sostanzialmente dalle ordinarie. Due doppie guide costituite da grosse lastre di vetro da specchi lunghe oltre 3 metri, spianate accuratamente sui bordi, sono poste ad angolo retto in un piano rigorosamente orizzontale. Su ciascuna doppia guida scorre una slitta metallica, portante una doppia piattaforma munita di viti micrometriche, di registro e di livello. Su una delle doppie piattaforme riposa il reticolo $\mathrm{R}$, sull altra il sistema oculare $O$ (fig. 1). Un tirante di ottone RO riunisce le slitte; 
nel suo punto medio $M$ è attaccata, in modo da essere facilmente registrata, una seconda asta MC rigida e leggiera che può scorrere colla sua estremitá, provvista di una punta, dinanzi ad un lungo telaio $\mathrm{T}_{1} \mathrm{~T}_{2}$, incurvato come la circonferenza di diametro Ro e poggiato su un cavalletto da fotogiafia.

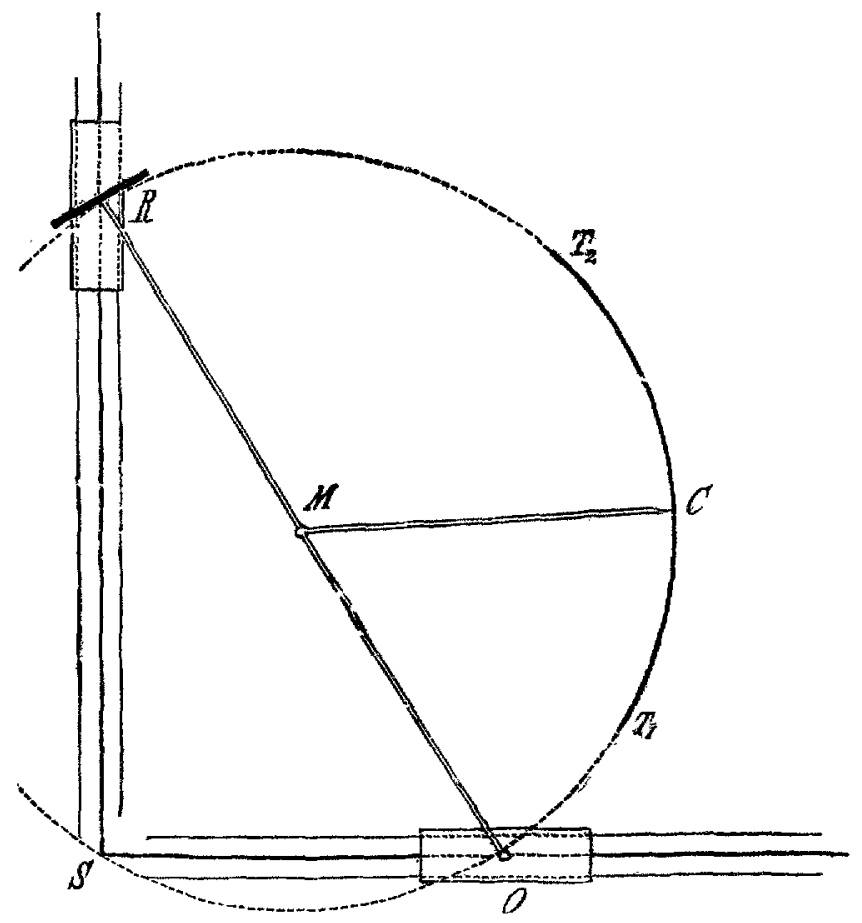

Fijg. 1.

Su una delle lastre di vetro è attaccata una scala graduata, sulla quale sono notate le lunghezze d' onda corrispondenti alle posizioni del reticolo e le distanze dell' oculare $O$ dalla fenditura, posta nel punto di incontro $\mathrm{S}$ delle linee mediane delle doppie guide.

3. Ialla teoria del reticolo è noto che con una tale disposizione si possono, al solo scorrere delle slitte, osservare gli spettri normali dei primi tre ordini, i quali si trovano ad ogni 
jstante situati sulla circonferenza di diametro RO. Ma poichè esiste luminositá in tutti quei punti per i quali si verifichi la relazione

$$
e(\operatorname{sen} i+\operatorname{sen} 9)=\mathrm{N} \lambda,
$$

fra la costante $e$, gli angoli $i$ e $\vartheta$ di incidenza e di diffrazione contati a partire dalla retta $R O$, l'ordine $N$ e la lunghezza d'onda $\lambda$, cosi gli spettri ora rammentati non sono i soli forniti dal reticolo. Altri se ne formano infatti dalla parte opposta, a destra - cioè - di chi guardi il reticolo, e precisamente fra questo e la imagine $S^{\prime}$ della fenditura regolarmente rifiessa (fig. 2).

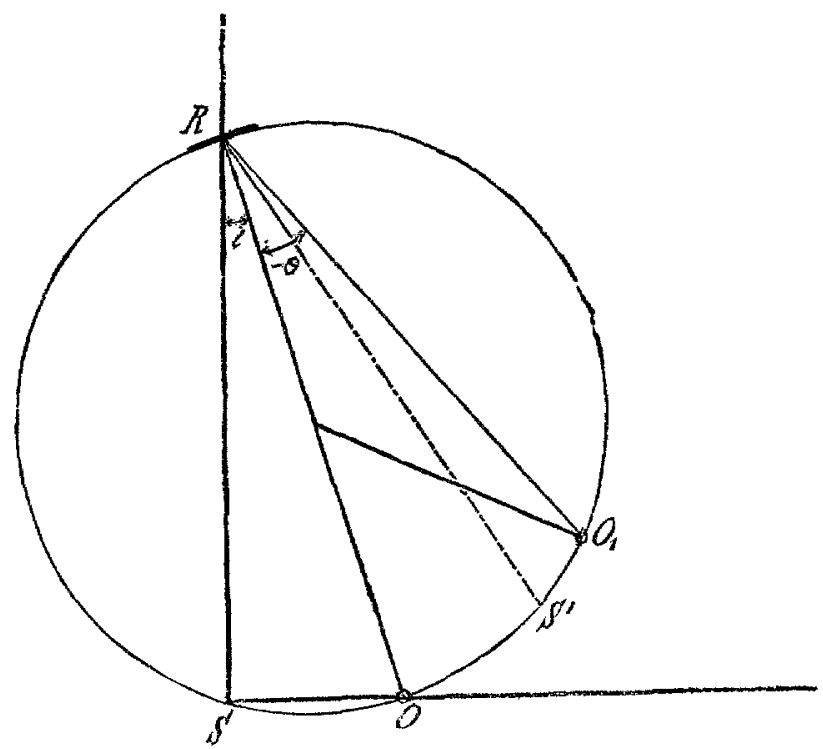

Fig. 2.

Per le proprietá fisiche, gli spettri situati a destra differiscono da quelli situati a sinistra per essere più alti, più luminosi e più dispersi di questi, come ha anche notato il Prof. Rizzo descrivendo una disposizione ${ }^{1}$ ) da lui creduta vantaggiosa per il loro. studio.

1) G. Rizzo, Atti Acc, di T'orino, Vol。 34. 
$\mathrm{E}$ infatti se si osserva che per questi spettri $i$ e 9 rjescono sempre di segno contrario, si-scorge subito dalla formola precedente che i successivi valori 9 corpispondenti ad una lunghezza d'onda fissa vanno aumentando in valore assoluto, col crescere dell' angolo $i$. E allora dalla formula

$$
\frac{d \vartheta}{d \lambda}=\frac{\mathrm{N}}{e \cos \vartheta}
$$

che esprime la dispersione in funzione di $\vartheta$, si ricava che anche la dispersione cresce con $i$.

Spostando quindi il reticolo da una posizione $\mathrm{R}_{1}$ ad un'altra $R_{2}$ per modo che sia $i_{2}>i_{1}$, si otterrá uno spettro ultravioletto del primo ordine $I_{2}$ più disperso per tutta la sua estensione e a parità di lunghezza d'onda, dello spettro $I_{1}$ corrispondente alla prima posizione. Aumentando notevolmente il valore dell' angolo d'incidenza, si potrà far raggiungere a tale spettro di destra del primo ordine una dispersione che si avvicini o superi quella posseduta dallo spettro normale del secondo ordine.

In ciò consiste appunto il metodo che ho seguito.

Quando tutta la montatura del reticulo è esatta, una pellicola stesa sul telajetto rigorosamente incurvato come la cir. conferenza di diametro RO corrispondente alla assunta posizione del reticolo è, diciamo così, anch' essa a foco e può ricevere l'impressione delle imagini spettrali. In un tale spettro, è vero, la dispersione non è costante, poichè essa cresce con $\lambda$; peró in molti casi ciò non costituisce un inconvenjente.

In queste fotografie riesce semplice anche il calcolo ed il riconoscimento delle lunghezze d'onda; raccogliendo sempre sulla stessa pellicola $\mathrm{I}^{*}$ imagine della fenditura regolarmente riflessa e lo spettro, se $s$ è la distanza fra una riga qualsiasi e quella imagine, per la precedente relazione (1) si ha, come è noto, facendo $\mathrm{N}=1$ :

$$
\lambda=2 e \operatorname{sen} \frac{s}{4 r} \cos \left(i-\frac{s}{4 r^{r}}\right)
$$

dove $\lambda$ è espresso per i valori di $s$ e di $i$ e $2 r=$ RO. 
SULL' USO DEL RETICOLO DI DIFFRAZIONE, EGC.

Le distanze $s$ si misurano sulle pellicole dopo che queste sono state sviluppate e asciugate. Quanto ai valori dell' incidenza $i$, essi si possono ricavare leggendo le distanze so (fig. 1) segnate sulla scala che, come ho già detto, è unita all'apparecchio. Se poi nell'oculare comparisce una riga la cui lunghezza d'onda sia conosciuta, allora l'angolo di incidenza sarà noto senz' altro, e con maggiore precisione, per mezzo della formola

$$
\operatorname{sen} i=\frac{\mathrm{N} \lambda}{e}
$$

Con questo metodo ho ottenuto delle fotografie di una nitidezza, di una intensità $e$ di una dispersione inaspettate e sono stato in grado di compiere degli studi, nei quali avrei dovuto necessariamente valermi dello spettro normale del secondo ordine.

Ho potuto cosi evitare gli inconvenienti presentati da quest' ultimo ed approfittare al tempo stesso di quella singolare luminosità che distingue lo spettro di destra del primo ordine. 